
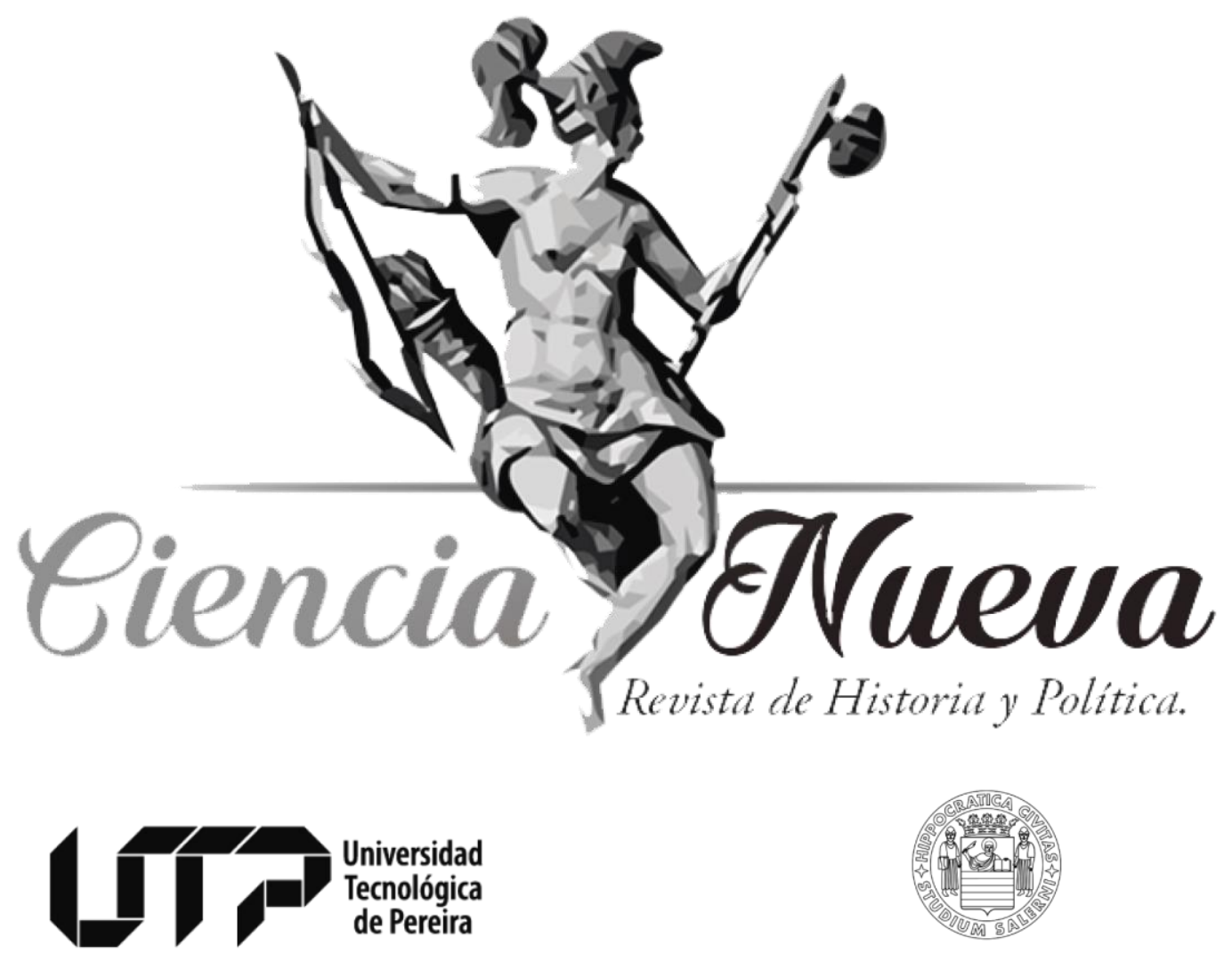

UNIVERSITÀ DEGLI STUDI DI SALERNO

Maestría en Historia

Maestría en Ciencia Política

ESTUDIOS HISTÓRICOS

\title{
EL SEMILLERO DE INVESTIGACIÓN COMO HERRAMIENTA DIDÁCTICA PARA LA FORMACIÓN DE SUJETOS CRÍTICOS DE \\ LA HISTORIA LOCAL
}

THE RESEARCH GROUP AS A PEDAGOGICAL TOOL TO TRAIN CRITICAL THINKING ABOUT

THE LOCAL HISTORY

DOI: https://doi.org/10.22517/25392662.20861

Sandra Catalina Clavijo Vélez

pp. 16-37

Vol. 3 Núm. 1 | Enero-Junio de 2019

Pereira, Colombia 


\section{EL SEMILLERO DE INVESTIGACIÓN COMO HERRAMIENTA DIDÁCTICA PARA LA FORMACIÓN DE SUJETOS CRÍTICOS DE LA HISTORIA LOCAL*}

\section{THE RESEARCH GROUP AS A DIDACTIC TOOL TO TRAIN CRITICAL THINKING ABOUT THE LOCAL HISTORY}

Sandra Catalina Clavijo Vélez.*

catalinaclavijov@gmail.com

ORCID: http://orcid.org/0000-0003-4265-9674

\begin{aligned} & \hline Recibido: 15 de octubre de 2018. \\ & Revisado: 27 de febrero de 2019. \\ & Aceptado: 11 de marzo de 2019. \\ & Publicado: 30 de junio de 2019. \\ & \hline\end{aligned}

\section{Resumen}

Este artículo describe la implementación de una Estrategia de Investigación Formativa con estudiantes de educación media en ArmeniaQuindío, durante el año 2017. Experiencia que permitió el acercamiento de los estudiantes a la historia de la configuración barrial de un asentamiento informal, por medio de un semillero de investigación formativa. En dicho escenario, se formó a los estudiantes en el oficio del historiador, las técnicas de investigación social, el análisis, elaboración y presentación del informe final de investigación, ante la institución educativa y la comunidad. Finalmente, los estudiantes reflexionaron sobre los impactos del Semillero como herramienta didáctica para la enseñanza de la Historia local.

\section{Palabras claves}

Semillero de investigación, historia local, educación media, investigación formativa, enseñanza de la historia

\begin{abstract}
:
This article describes the implementation of a formative research strategy, with high school students in Armenia, Quindío, during 2017. This experience allowed to get the students close to the history of the configuration of an informal neighborhood settlement, through a formative research group. In this scenery, the students were taught into the job of the historian, the social research techniques, and the analysis, elaboration and presentation of the final research report to the educational institution and the community. Finally, the students thought critically about the impacts of the research group as a didactical tool to teach the local history.
\end{abstract}

\section{Keywords:}

Research group, local history, formative research, middle education, history teaching.

\footnotetext{
* Este artículo de investigación se deriva del proyecto de mejoramiento institucional denominado "El semillero de investigación como herramienta didáctica para la formación de sujetos críticos de la historia local", desarrollado como tesis de grado para optar por el título de magister en Historia de la Universidad Tecnológica de Pereira, en el marco del Programa - Becas para la Excelencia Docente-, del Ministerio de Educación Nacional. Este artículo respeta las directrices y normas dispuestas en la Declaración de Ética de Publicación de Ciencia Nueva, Revista de Historia y Política. Esta declaración puede consultarse en la página web de la revista: revistas.utp.edu.co/index.php/historia.

** Trabajadora social de la Universidad del Quindío y candidata a Magíster en Historia por la Universidad Tecnológica de Pereira. Docente de Ciencias Sociales de la Institución Educativa República de Francia, Armenia, Quindío.
} 


\section{Introducción}

$\mathrm{E}$ n la educación básica, secundaria y media colombiana es pertinente dar vía libre a propuestas que incluyan el estudio y análisis de la historia desde sus diferentes corrientes y métodos (innovando en didácticas y abordajes); promover la praxis: acompañar la teoría e información suministrada en clase con una práctica investigativa concreta, en la que los estudiantes experimenten, indaguen, obtengan, contrasten y lleguen a conclusiones por sí mismos, desarrollando un proyecto investigativo puntual sobre un espacio concreto, vivo, donde hay personas que guardan en la memoria (en archivos improvisados, en fotos y relatos) información relevante, no solo sobre una genealogía propia sino también sobre la historia de la conformación barrial y local.

Estas afirmaciones se argumentan con lo siguiente: en Colombia, la enseñanza de las ciencias sociales en la escuela, según la Ley General de Educación de 1994, se centra principalmente en las disciplinas de historia y geografía ${ }^{1}$. A su vez, esta ley deja entrever la necesidad de contar con ciudadanos participativos y críticos comprometidos con lo público.

Por otra parte, uno de los fines y objetivos de la educación en Colombia, planteado desde los lineamientos curriculares del área de ciencias sociales busca "Ayudar a comprender la realidad nacional (pasado-presente) para transformar la sociedad en la que las y los estudiantes se desarrollan, donde sea necesario"2.

Por esta razón es importante apostarle a un mejoramiento de la comprensión de diversos hechos sociales e históricos por parte de los estudiantes. No solo apelando a fechas, sino a los antecedentes, causas, y profundas consecuencias de algunos procesos o momentos en la historia y cómo estos generaron transformaciones y repercuten hoy en nuestro contexto. Al respecto, el Ministerio de Educación Nacional expresa que:

(...) durante la Educación Básica y Media es importante y necesario que se forme en los conceptos básicos y se practiquen métodos y técnicas propios de las diversas disciplinas que conforman las Ciencias Sociales. Por ejemplo, un educando que curse la básica y la media no será un historiador, pero debe aprender a manejar y seleccionar fuentes, que es algo básico en el conocimiento histórico ${ }^{3}$.

Es así como este artículo sistematiza la experiencia del Semillero de Investigación "Cultivando Historia" desde su proceso de conformación, pasando por la etapa teóricaformativa, el trabajo de campo, el análisis de los hallazgos, la socialización de resultados y los aprendizajes. Escenario que fue asumido como una estrategia didáctica y un espacio de encuentro y producción de conocimiento, para hacer una aproximación a la historia local a través del estudio del barrio de los estudiantes investigadores.

\section{Problema de investigación}

Frente al panorama actual de la educación básica y media en Colombia, donde no se le da el lugar que merece a una disciplina tan importante como la historia, y su construcción de

\footnotetext{
${ }^{1}$ Ley 115/1994, del 8 de febrero. Ley general de educación. Diario Oficial No. 41.214. República de Colombia. https://www.mineducacion.gov.co/1621/articles-85906_archivo_pdf.pdf.

${ }^{2}$ Ministerio de Educación Nacional, “Lineamientos curriculares Ciencias Sociales” (2002), 13. https://www.mineducacion.gov.co/1621/articles-89869_archivo_pdf.pdf.

${ }^{3}$ Ministerio de Educación, "Lineamientos curriculares Ciencias Sociales”. .., 13.
} 
perspectivas emancipadoras, críticas y comprensivas del pasado para interpretar el presente, somos los docentes quienes debemos preguntarnos: ¿Hacia dónde va la enseñanza de las ciencias sociales? Pero, aunque las críticas son bienvenidas, precisamos propuestas.

Resulta necesario lo que Lefebvre llamaba una "perspectiva desde abajo" para ver la historia y entenderla en contraste con las clases populares. Lo anterior para oxigenar un currículo que actualmente reproduce, pero no transforma los conceptos y didácticas de disciplinas como la historia. Su valor debe estar pensado no solo desde lo cognitivo, sino desde el impacto en la comunidad, la reivindicación de derechos y el poder de incidir en la realidad social de los estudiantes como actores, donde prime la dignidad humana. La educación debe dar herramientas para la vida, debe derivar en perspectivas y miradas humanistas de la sociedad de la cual se es parte.

La educación contemporánea debe reconocer cada vez más la interacción y el papel del estudiante. La preocupación no debe ser trasmitir información. Los estudiantes no son "receptores" así los conceptos estarían condenados al olvido. Estos patrones no han tenido los mejores resultados, y para comprobarlo basta mirar el abanico social, los miles de graduados del pasado que han pasado a engrosar las filas de los oprimidos, de los sin techo, de los que aguantan cualquier exabrupto y corruptela estatal. Al respecto, Carr (1961) afirma que:

La historia consiste esencialmente en ver el pasado por los ojos del presente y a la luz de los problemas de ahora, y que la tarea primordial del historiador no es recoger datos sino valorar: porque si no valora ¿cómo puede, saber lo que merece ser recogido? ${ }^{4}$.

La investigación en ciencias sociales e historia otorga la posibilidad de cuestionarse constantemente y plantearse preguntas que de otra forma no surgirían, brinda la posibilidad de estar en contacto con el otro, desarrolla la empatía, el diálogo de saberes y aviva el deseo de comprender la realidad. Teniendo siempre, por supuesto, una perspectiva horizontal y participativa en la construcción de conocimientos socialmente relevantes.

Por esta razón se plantea la siguiente pregunta de investigación: ¿Cómo hacer de la enseñanza de la historia una experiencia que permita a los estudiantes cuestionar e interpretar su presente y su entorno, a través del conocimiento de los hechos del pasado, y ser ciudadanos críticos con posturas claras sobre la identidad, la memoria y la historia local?

El objetivo general de esta propuesta fue diseñar e implementar una estrategia de investigación formativa, para el acercamiento a la historia del asentamiento informal Salvador Allende, de la ciudad de Armenia (Quindío); por medio del Semillero de Investigación Cultivando Historia del grado 10-A de la Institución Educativa República de Francia, durante el año 2017.

Adicional a ello, se formó a los estudiantes en los métodos de investigación social y en el oficio del historiador, aplicaron técnicas de investigación que les permitió dar cuenta de la historia de su barrio. Analizaron la información, elaboraron y presentaron el informe final de investigación, ante la comunidad educativa y la Junta de Acción Comunal (JAC) del asentamiento objeto de estudio. Por último, el docente reflexionó sobre los impactos del

\footnotetext{
${ }^{4}$ Edward Hallet Carr, ¿Qué es la historia? (Conferencias, "George Macaulay Trevelya" dictadas en la Universidad de Cambridge. Enero - marzo de 1961) (Universidad de Cambridge: Editorial Ariel, S.A.), https://introduccionalahistoriaunlp.files.wordpress.com/2014/03/carr-que-es-la-historia-cap1-el-historiador-ylos-hechos.pdf.
} 
semillero de investigación como herramienta didáctica para la enseñanza de la Historia y las ciencias sociales en educación media.

En esta propuesta confluyeron marcos teóricos, metodológicos y prácticos de las didácticas de las ciencias sociales, la historia, la historia local y barrial; la historia desde abajo; el rescate de la memoria; el acercamiento de los estudiantes al conocimiento como científicos sociales; la orientación en temas disciplinares, principalmente, la formación y generación de pensamiento crítico para que los actores de la experiencia lograran apropiarse y producir aprendizajes y conocimientos socialmente relevantes, reflexionar el pasado en clave del presente y llevar a cabo acciones transformadoras en su entorno y comunidad.

\section{Investigación formativa}

El semillero de investigación es un escenario sui generis, es diferente a aquel grupo de estudiantes que recibe clases regulares en el colegio, algunas de ellas con 40 estudiantes o más por aula, donde la calidad y la enseñanza personalizada riñen con la cobertura y la reducida cantidad de docentes. Sumado a esto, en dichos grupos regulares, muchas veces el docente además de lo académico es responsable de recibir y direccionar información sobre distintas problemáticas familiares, del entorno, económicas y sociales, que dificultan el proceso de enseñanza y aprendizaje de los jóvenes. En este sentido, los grupos pequeños de trabajo, como los semilleros de investigación, permiten profundizar en los temas, conocer mejor a los integrantes del mismo y generar cierta empatía e identidad por el colectivo y su trabajo.

Por otro lado, desde la experiencia del Semillero de Investigación Cultivando Historia se considera que la investigación formativa, la pedagogía crítica, la historia desde abajo, entre otros, pueden ser caminos diversos para la producción de conocimiento. Desde esta perspectiva no se trabaja con las comunidades como sujetos pasivos, sino como agentes activos de la transformación, en tanto se recupera colectivamente la historia de forma participativa, donde cada uno se reconoce como actor. Sobre este tema, Sánchez menciona:

A investigar se aprende aliado de otro más experimentado; a investigar se enseña mostrando cómo; a investigar se aprende haciendo, es decir, imitando y repitiendo una y otra vez cada una de las complejas y delicadas labores de la generación de conocimiento; a investigar se enseña corrigiendo; se aprende viendo 5 .

\section{¿Por qué enseñar historia desde abajo?}

Porque abajo están las bases, porque abajo están los relatos fundacionales, porque abajo es donde ocurre la vida real y abajo está la mayoría, quienes transforman el lenguaje y le dan impulso al sistema. Por ello, si logramos cambiar la percepción de los subalternos, respecto a su lugar de enunciación, podremos transformar el orden de las cosas.

\footnotetext{
${ }^{5}$ Ricardo Sánchez Puentes, Enseñar a investigar: Una didáctica nueva de la investigación en ciencias sociales y humanas (Universidad Nacional Autónoma de México: Instituto de Investigaciones sobre la Universidad y la Educación, 2014), 14-15, http://disde.minedu.gob.pe/bitstream/handle/123456789/4635/Ense\%C3\%B1ar\%20a\%20investigar\%20una\% 20did\%C3\%A1ctica\%20nueva\%20de\%20la\%20investigaci\%C3\%B3n\%20en\%20ciencias\%20sociales\%20y $\%$ 20humanidades.pdf?sequence=1\&isAllowed $=\mathrm{y}$.
} 
Para Torres «"abajo" y "sur" no son solo expresiones espaciales, sino políticas, acuñadas desde diferentes lugares (literarios, investigativos, conceptuales) y luchas que develan opresiones y exclusiones presentes en diferentes ámbitos de la vida social» ${ }^{6}$.

Existe un amplio acervo de fuentes que pueden brindar información sobre la historia desde abajo, aquella que las comunidades pueden contar con autoridad a partir de sus vivencias, sus luchas y sus logros. Un ejemplo de ello, es la constitución de un barrio informal, con todas las particularidades y dificultades que conlleva este proceso. Además del trabajo colectivo en torno a la construcción de comunidad. La historia desde abajo, empodera a las personas del común para que puedan hacer uso de la palabra y a través de la narrativa exponer sus experiencias en torno a búsquedas colectivas y particulares, constituyentes del entramado que conforma la sociedad y la historia general.

Además, se tiende un puente entre los intereses de los estudiantes y los intereses de la disciplina histórica. Desde el microcosmos que puede representar un barrio o una localidad, es posible hacer lecturas amplias de la realidad, el presente, el pasado y hasta elucubraciones sobre el futuro. La comunidad se convierte en un excelente caldo de cultivo, de cual no solo se toma, sino, por el contrario, se requiere participación plena para construir conjuntamente y visibilizar problemáticas y gestionarlas. Por otro lado, este proceso se acompañó de la historia oral, que según Thompson:

(...) es un método que siempre ha sido esencialmente interdisciplinario, una especie de cruce de caminos entre sociología, la antropología, la historia y los análisis literarios y culturales (...) la fuerza crucial de la historia oral descansa sobre una forma fundamental de interacción humana que trasciende fronteras disciplinarias ${ }^{7}$.

La historia debe permitir a los estudiantes un acervo conceptual y una base teórica sobre la cual elaborar procesos de pensamiento para la toma informada y formada de decisiones, desde posturas críticas, que no solo permitan rumiar las situaciones sociales, sino apropiarse de ellas y sobre todo, actuar.

\section{Metodología}

\section{Implementación de la propuesta didáctica}

El proyecto de investigación formativo tuvo como escenario inicial la clase de Ciencias Sociales, con 20 estudiantes del grado 10-A de la Institución Educativa República de Francia. De igual forma, se conformó de manera paralela el Semillero de Investigación Cultivando Historia (SEICHI), constituido por 7 estudiantes de los grados décimo y noveno de la institución.

Así se complementaron dos momentos: el primero de formación teórica y conceptual en el aula, y el segundo, la participación como agentes claves en la ejecución del trabajo de campo, contrastando la teoría y la realidad. "Aprender haciendo".

La propuesta tuvo incidencia en la reestructuración del plan de área de Ciencias Sociales en lo que respecta al grado décimo, que durante el segundo periodo tendrá en adelante un tópico generativo específico para el estudio de la historia local y la investigación

\footnotetext{
${ }^{6}$ Alfonso Torres Carrillo, Hacer historia desde abajo y desde el sur (Bogotá: Ediciones desde abajo, 2014), 1, https://www.academia.edu/32287760/Hacer_historia_desde_abajo_y_desde_el_Sur.

${ }^{7}$ Paul Thompson, "Historia oral y contemporaneidad", ANUARIO N ${ }^{\circ} 20$ - Escuela de Historia - FH y A - UNR (2017), www.anuariodehistoria.unr.edu.ar/ojs/index.php/Anuario/article/download/204/223.
} 
social e histórica. Además, se articuló con el Proyecto Institucional de Democracia. Lo cual responde al Proyecto de mejoramiento institucional, de acuerdo a lo requerido por el Ministerio de Educación Nacional en el programa Becas para la Excelencia Docente.

\section{Primer momento}

En la institución educativa el área de Ciencias Sociales tiene asignadas cuatro horas a la semana, que se debían distribuir entre 7 materias que constituyen el área. En este sentido, se utilizaron 3 horas semanales para el proyecto de historia local, esto para abordar todos los contenidos disciplinares necesarios, hacer la apropiación de conceptos y hacer ejercicios y pruebas piloto con todos los estudiantes del grado décimo en el aula.

\section{Tópicos generativos trabajados:}

- Hacer historia desde abajo y desde el sur, Alfonso Torres Carrillo.

- ¿Para qué historiar? La función social de la historia, Joaquím Prats.

- Técnicas de investigación social e histórica. La caja de herramientas del joven investigador, Jocelyn Létourneau.

- La Investigación Formativa, Bernardo Restrepo Gómez.

- "La Cartografía Social" ejercicio de levantamiento de información en el colegio.

- Análisis de los videos: discurso para los estudiantes, Salvador Allende; La Oralidad en la investigación histórica y Moravia memoria de resistencia.

- Elaboración del instrumento de investigación y pruebas piloto.

\section{Segundo momento:}

Éste consistió en la conformación del Semillero de Investigación Cultivando Historia. A partir de este espacio de aprendizaje se llevó a cabo con los estudiantes una aproximación a la historia del barrio donde muchos de ellos habitan actualmente, y que a su vez hace parte del sector de influencia de la institución educativa.

Con la conformación de este colectivo se inició la ejecución del trabajo de campo en el asentamiento informal Salvador Allende, a través de reuniones extraclase durante dos días a la semana, con una intensidad de tres horas en cada encuentro.

Así, los estudiantes efectuaron el levantamiento de información a través de diferentes técnicas de investigación social para la recuperación de la memoria, por medio de la historia oral, de documentos de archivo sobre el barrio, periódicos locales, fotografías antiguas de los pobladores, documentos notariales, mapas físicos y satelitales, tesis de grado, libros sobre la reconstrucción del eje cafetero, documentos oficiales de la administración municipal y la JAC del asentamiento.

Acciones:

- Recorridos por el barrio, habitar el territorio.

- Entrevistas y reuniones con líderes comunitarios.

- Levantamiento de archivo fotográfico antiguo y actual del lugar.

- Visitas a centros de documentación.

- Participación en la vida comunitaria del asentamiento.

- Elaboración de crónicas.

- Realización de tomas para el informe final y video. 
Este proyecto se articuló a su vez con el modelo pedagógico del colegio, Enseñanza para la Comprensión ${ }^{8}$, al tener aspectos similares compartidos, como las preguntas y presaberes de los estudiantes (base del nuevo conocimiento) y un lugar importante para la investigación, especialmente en la evaluación, donde se orienta al estudiante en tres etapas: exploración, investigación guiada y proyecto final de síntesis, proceso similar al trabajo hecho en un semillero, con el valor agregado del trabajo fuera del aula (Figura 1).

\section{Etapa de exploración}

Aquí se establecen relaciones entre presaberes, intereses propios de los estudiantes y tópicos generativos planteados en el área.

\section{Investigación guiada}

Busca comprometer y vincular a los estudiantes en procesos de investigación para producir conocimiento, ampliar y complementar tópicos y conceptos trabajados en clase.

\section{Proyecto final de síntesis}

Producción intelectual y/o artística independiente por parte del estudiante que sintetiza la comprensión del tópico y los conceptos estudiados durante un periodo.

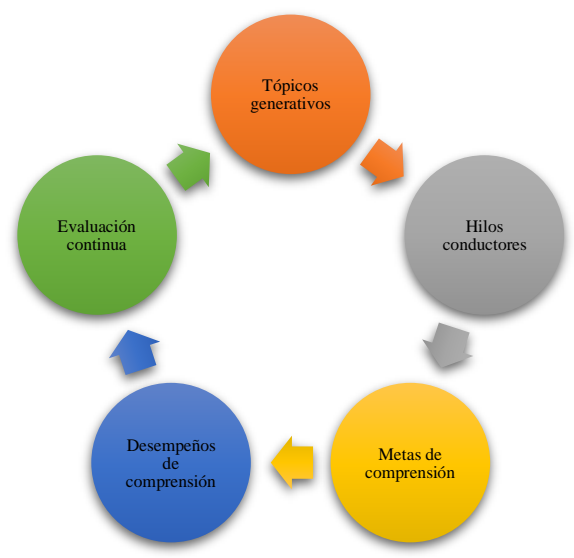

Figura 1. Modelo Enseñanza para la Comprensión (EpC).

\section{Metodología del proyecto de investigación}

\section{Tipo de estudio}

El abordaje del contexto local, como aproximación a la historia del asentamiento informal Salvador Allende de la ciudad de Armenia, en términos físicos y sociales se planteó como una investigación de tipo descriptivo.

\section{Método}

El diseño de la investigación se apoyó en el método cualitativo porque buscó (desde la historia oral, a través de narraciones de fuentes vivas) que los estudiantes hicieran una aproximación sistemática a la historia del asentamiento informal Salvador Allende de Armenia.

\section{Técnica de recolección de datos}

Los datos fueron obtenidos mediante la aplicación de la técnica de la entrevista, cuyo instrumento contiene 40 preguntas, y se estructuró con una división por categorías de análisis o secciones, así:

- Identificación general y origen del asentamiento.

- Infraestructura del sector.

- Características demográficas y sociales.

\footnotetext{
${ }^{8}$ Para ampliar este tema ver: "Enseñanza para la Comprensión (Tomado de ALPS de la Universidad de Harvard en http://learnweb.harvard.edu)", SCRIBD, acceso el 27 de mayo de 2019, https://www.scribd.com/document/336645771/Comprension-Metas-Desempenos-Valoracion.
} 
- Características culturales.

- Equipamientos comunitarios, organización social y participación.

Por otra parte, se utilizó la observación participante en diversos recorridos por el territorio y se realizaron algunos ejercicios de cartografía social para complementar la información de primera mano. Finalmente, se consultaron fuentes secundarias.

\section{Población y muestra}

La población o universo de estudio ${ }^{9}$ fueron 979 habitantes del asentamiento informal Salvador Allende. Dichas personas residen en 267 viviendas, según el Diagnóstico Local Participativo de $2016^{10}$.

Inicialmente en el aula se realizó una aproximación crítica a los aspectos teóricos de la historia, tales como su origen, definición y representantes; la diferencia entre memoria, recuerdo, historia personal e historia general; los conceptos claves de esta disciplina; las fuentes utilizadas; las técnicas de investigación existentes; las corrientes a lo largo de su acontecer (especialmente la historia nueva) y la periodización.

Respecto a los archivos como forma de conocer los hechos históricos, particularmente los del barrio estudiado, se tuvo acceso a fotografías, recortes de prensa, escrituras, mapas y los relatos de los vecinos.

En síntesis, se vivieron dos momentos: el primero responde al abordaje teórico por parte del docente y la transposición didáctica de estos contenidos con los estudiantes en el aula. El segundo, la práctica asumida desde la simulación del oficio del historiador (como estrategia didáctica) en campo. En ella fueron claves el trabajo responsable, autónomo y colaborativo. Este proceso no se centró únicamente en contenidos, se valoró también la experiencia investigativa directa con fuentes, personas, vías y caminos. Todo con el fin de lograr la resignificación del espacio urbano ${ }^{11}$.

\section{Resultados}

El análisis e interpretación de la información incluyó un abordaje del contexto social, las generalidades del asentamiento informal, la elaboración de productos audiovisuales y la socialización de hallazgos con la comunidad. También, la reflexión docente respecto al impacto del proceso llevado a cabo con los estudiantes, la comunidad y la institución, así como sus consecuencias en cada uno de los actores. Finalmente, se detallaron los logros y problemáticas encontradas en el camino, los aprendizajes y las conclusiones de esta búsqueda.

\footnotetext{
${ }^{9}$ Los informantes claves fueron identificados con la asesoría de la JAC. Entre ellos, los habitantes con mayor antigüedad de residencia en el sector, líderes, posibles fundadores, primeros habitantes y familiares de los estudiantes del semillero con amplio tiempo de residencia en el lugar.

10 Departamento Administrativo de Planeación, Diagnóstico Local Participativo. CODELPA. Comité de desarrollo local participativo de Armenia (2016), http://planeacionarmenia.gov.co/comuna-10/

${ }^{11} \mathrm{El}$ asentamiento informal Salvador Allende es uno de los más grandes de la ciudad, se encuentra en la periferia de la zona norte de Armenia, paralelo a la vía que conduce a Pereira en la zona del Pórtico. Es invisible en el paisaje urbano, a diferencia de la informalidad en otras zonas del país que se evidencia en las lomas de las ciudades, en Armenia esta se encuentra restringida y oculta en el interior del relieve, cerca de quebradas y laderas, en este caso, sobre la antigua vía férrea que comunicaría a Armenia con Ibagué.
} 


\section{Productos y hallazgos de la historia barrial}

¿Por qué seleccionar el barrio como objeto de estudio de la historia local, y no los pocos monumentos o lugares históricos, los personajes fundadores de la ciudad o la llegada de los colonizadores y el camino del Quindío? Porque según Correa:

La elección del barrio como unidad de análisis socio-espacial o como laboratorio microsocial desde una perspectiva histórica, facilita el seguimiento de la pista de una serie de diferentes procesos sobre los cuales se ha tejido, quizás de manera muy inestable, un tipo específico de sociedad barrial, una sociedad territorializada, de muros invisibles, dentro de una sociedad mayor que sería el conjunto de la sociedad urbana ${ }^{12}$.

El barrio, ese pequeño cosmos, brinda la posibilidad de encontrar vestigios del pasado y dar rienda a otras preguntas de corte histórico más amplias. En él se conjuga la vida a pequeña escala y es posible extrapolar diferentes temas (económicos, políticos, culturales y sociales) y compararlos con otros contextos más amplios, la ciudad, el departamento, la región y el país.

Además, es la patria chica de los jóvenes, allí están los orígenes de su vida (la piel, la casa y el barrio son nuestras primeras capas protectoras constitutivas). La investigación requiere, ante todo, iniciarse con un tema donde se parta de una vivencia personal, una búsqueda visceral o un pasado propio, solo así habrá un catalizador de nuevas preguntas en el futuro.

\section{Algunas apreciaciones sobre el asentamiento informal Salvador Allende}

$\mathrm{Al}$ recorrer a pie el lugar, se observa que el espacio y sus pobladores aún conservan una marcada estética y prácticas rurales. Posee una sola calle vehicular, al lado de la cual se erigen las viviendas; esta configuración se debe a que el barrio se construyó sobre la vía férrea, razón por la cual es una larga y estrecha franja que no ha tenido mayores posibilidades de ampliación hacia los lados.

Su origen coincide con los procesos de migración del campo a la ciudad de los años 50 y 60. La mayoría de pobladores provienen de zona rural de los municipios de la cordillera quindiana. Se identificó población indígena originaria de resguardos de Riosucio (Caldas), algunos de ellos desplazados por factores de violencia en la zona.

Los materiales de las viviendas son una amalgama de elementos transitorios y permanentes. La informalidad se manifiesta tanto en las formas de vida como en los oficios de los pobladores (responden a las lógicas del rebusque), pues la formación académica de la mayoría de la población adulta es básica.

Según una investigación adelantada por Clavijo, en términos de las características físicas de los asentamientos informales en Armenia, se encontró que en dichas zonas:

El 47\% (941 unidades) de las viviendas fueron construidas por sus propietarios, mientras que el 14\% (270 unidades) se construyeron con la ayuda de personas capacitadas en el tema. Por su parte, la opción de la minga o el convite denota un bajo porcentaje, tan solo un 5\% (108 unidades) de las personas que viven en estos sectores acuden a sus redes sociales de

\footnotetext{
12 Jhon Jaime Correa Ramírez, "Historia local: el ritmo de la historia barrial”, Antropología y Sociología: Virajes (2006). (Artículo de investigación. I Foro Regional de Historia Contemporánea, en la Universidad de Antioquia, 28 de octubre de 2005).
} 
intercambio a la hora de solucionar el problema de construcción de la vivienda. En cuanto al $34 \%$ (682 unidades) restante, equivale a viviendas que fueron adquiridas total a parcialmente terminadas, a familias arrendatarias o a familias que al momento de responder la encuesta ignoraban la forma de construcción ${ }^{13}$.

Respecto a los primeros materiales utilizados por los habitantes del lugar en la construcción de las viviendas, se identificó principalmente el uso de guadua y esterilla. Sobre la escolaridad de los pobladores, Ramírez afirma que:

Según la teoría del capital humano la educación eleva la eficiencia de las personas en la producción de bienes y servicios, y se traduce además en mayor nivel de ingresos de la persona educada; pero al mismo tiempo, le proporciona al individuo otros beneficios, como poder leer, comunicarse y desarrollar otras actividades que contribuyen a hacerlo más humano ${ }^{14}$.

En este sentido, el nivel de escolaridad de los jefes de familia determina el ingreso económico que tienen actualmente los hogares, bastante precario por demás en las zonas informales de la ciudad. En el barrio, la mayor parte de los jefes de hogar se desempeñan en trabajos por cuenta propia (comerciantes, vendedores, indigentes, lavadores de carros, vigilantes independientes, carretilleros, etc.). También hay obreros, empleadas domésticas y desempleados.

Hay una alta vulnerabilidad social y económica en los pobladores de la zona. Si bien con los años esta ha tenido un paulatino proceso de consolidación física, representada en el mejoramiento de los materiales de construcción de la vivienda y el acceso a servicios públicos domiciliarios, hechos como el terremoto y el proceso de reasentamiento adelantado por el FOREC no generaron cambios significativos en el asentamiento Salvador Allende. Aunque fueron entregados algunos subsidios de vivienda, las cifras de la población que compone el barrio permanecen iguales.

Ahora bien, por el sector se calculó el paso del tren a partir de la construcción del ferrocarril Armenia-Ibagué. Al respecto, Martínez narra en Arrierías Salentinas, que esta obra:

Se consideró vital para el desarrollo del país y aún para la seguridad nacional. Su construcción se inició durante la presidencia del Señor Miguel Abadía Méndez (1926 - 1930). Una parte importante de los 20 millones de dólares que los Estados Unidos entregaron a Colombia como indemnización por el raponazo de Panamá, se gastó en las obras que se alcanzaron a construir desde entonces hasta 1948, año en el cual, el gobierno de Mariano Ospina Pérez, por múltiples razones, todas ellas insuficientes, suspendió de manera definitiva la construcción. Entonces la línea había avanzado desde Armenia hasta Boquía y desde Ibagué hacia Cajamarca, varios kilómetros ${ }^{15}$.

\footnotetext{
${ }^{13}$ Sandra Catalina Clavijo Vélez, "Características físicas y sociales de los asentamientos informales de Armenia en los años 1997- 2008” (tesis de pregrado. Universidad del Quindío, 2008), 145.

${ }^{14}$ Duván Emilio Ramírez Ospina, "Capital humano como factor de crecimiento económico: caso departamento de Caldas (Colombia). 1983 2003", acceso el 15 de mayo de 2019, http://www.eumed.net/librosgratis/2007b/271/4.htm.

15 Hernán Martínez Rincón, "El puente de Boquía", acceso el 15 de mayo de 2019, http://academiadehistoriadelquindio.blogspot.com/2014/07/el-puente-de-boquia.html. (Tomado de Arrierías Salentinas. Edición No. 2. Academia de Historia del Quindío, 2).
} 
Pero los rieles fueron levantados sin que se hubiese cumplido el objetivo. Con el tiempo llegaron a las casas de algunos pobladores antiguos, por parte de Ferrocarriles Nacionales de Colombia, las citaciones para organizar y legalizar la problemática incipiente de la posesión de tierra. Cita que únicamente cumplió don Juan Motato, pero solo para confirmarles a los funcionarios que él no abandonaría su casa, pues había comprado su terrero al dueño de la finca la Mariela, bajo la figura de loteo.

Las primeras tres casas del lugar se construyeron a mediados de la década del 50 y sus moradores habitaban en calidad de tenedores. Otros afirmaron haber comprado sus lotes a los dueños de las fincas existentes en la zona, entre ellas la Mariela. Nombre que posteriormente tomaría un barrio de la reconstrucción, ubicado en los terrenos que constituían esta finca y que limita con el asentamiento informal Salvador Allende.

En el asentamiento hay una fuerte presencia de población indígena, proveniente en su mayoría de resguardos de Riosucio (Caldas). Por esta razón, se entrevistó a don Sixto Gañan, quien fue gobernador indígena del cabildo embera chamí del Barrio Salvador Allende. Con tristeza en la voz, narró la pérdida de la lengua materna y mencionó que sería maravilloso contar con tierra para continuar las tradiciones de sus ancestros.

El asentamiento fue conocido inicialmente como La Carrilera, pero, a raíz de la muerte de Salvador Allende, uno de los líderes del sector decidió darle ese nombre al barrio, teniendo en cuenta la influencia que él había tenido en el pueblo latinoamericano en general. Para ello, realizaron gestiones con Ancizar López López con el fin de que se le otorgara legalmente ese nombre al lugar.

Las personas encuestadas tienen un sentimiento de arraigo generalizado hacia el territorio. Todas coinciden en que el barrio tiene mala fama, pero que realmente no la merece, pues no es peligroso. Este estigma se evidenció porque al lugar no envían domicilios de restaurantes o droguerías ni servicio de taxi.

Desde el Semillero de Investigación Cultivando Historia, se considera que los proyectos de investigación pueden contribuir al mejoramiento de la imagen de los barrios estudiados, lugares que en el pasado tuvieron problemas de seguridad y que han quedado en el imaginario colectivo pueden ser vistos con otros ojos a partir de la investigación histórica.

\section{Análisis de las variables estipuladas en el instrumento de investigación (entrevista)}

\section{La vida cotidiana en el asentamiento informal}

En esta comunidad existen redes sociales de apoyo. No solo son un grupo de personas que habitan un territorio, sino que comparten diferentes ámbitos cotidianos, se conocen y se apoyan en situaciones difíciles. Actitud que fortalece los lazos afectivos ante aspectos cotidianos como los nacimientos, la enfermedad, las celebraciones y la muerte. Aún perviven prácticas como velar a los difuntos en sus casas, escenarios donde los vecinos se reúnen a recordar al fallecido y a comentar sobre los pormenores de la vida del lugar.

También se identificaron pugnas, especialmente entre grupos generacionales. El barrio es un territorio donde cada uno busca hacerse a una identidad y albergan intereses particulares o colectivos diferenciados. En ocasiones, hay rupturas frente a proyectos que se pensarían de interés general, tales como la Junta de Acción Comunal (que se ha visto atravesada por redes clientelares a lo largo de su existencia, debido a estilos de liderazgo tradicionales que avalaron estas prácticas). 
A través de documentos fotográficos antiguos fue posible percibir la incidencia del poder político en el asentamiento (fiestas comunitarias, celebraciones a niños y ancianos, entregas de regalos y beneficios). La falta de voluntad política ha detenido en el tiempo el progreso del barrio, aun hoy se presentan allí candidatos a cargos públicos que venden la esperanza de la escrituración.

\section{Construyendo la identidad barrial}

Para contextualizar el ejercicio que se llevó a cabo desde el semillero de investigación, fue importante la descripción del asentamiento y comprender cómo en este espacio se construye la identidad barrial.

(...) los barrios, más que una fracción o división física o administrativa de las ciudades, son una formación histórica y cultural que las construye; más que un espacio de residencia, consumo y reproducción de fuerza de trabajo, son un escenario de sociabilidad y de experiencias asociativas y de lucha de gran significación para comprender a los sectores populares citadinos. En fin, los barrios populares son una síntesis de la forma específica como sus habitantes, al construir su hábitat, se apropian, decantan, recrean y contribuyen a construir cultura y políticas urbanas ${ }^{16}$.

En este sentido, cobró importancia la cartografía social como herramienta de investigación social, la cual permitió a los estudiantes apropiarse simbólicamente del territorio, darle sentido y pensarlo colectivamente, creando, a su vez, estrategias para transformarlo de manera positiva.

Año de fundación: doña Blanca González contó que el asentamiento apareció con la llegada de su familia al lugar, en 1955 (aseguró haber nacido allí). Así, las primeras viviendas se habrían construido hace más de 60 años. Ella nos narró la historia del barrio haciendo alusión a las personas fundadoras y a sus descendientes, así los estudiantes ubicaron y relacionaron a los primeros pobladores con personas que ellos conocen en la actualidad.

Por ejemplo, los Motato son una familia muy numerosa y conocida en el barrio y por ende hay muchos niños que estudian en el colegio y tienen ese apellido. Así mismo, logramos reconocer otros apellidos indígenas provenientes de familias de Riosucio como los Bueno, los Morales y los Gañan, que hacen parte del cabildo embera chamí de Salvador Allende.

Servicios públicos: actualmente el asentamiento cuenta con acueducto, alcantarillado, energía eléctrica, alumbrado público y recolección de basuras, algunas viviendas poseen instalación de gas domiciliario. Los primeros servicios empezaron a ser instalados a finales de la década del 80. Antes de eso, la población recurría al nacimiento de agua que se había adecuado en el lugar y tenía dos lavaderos comunitarios, para proveerse de luz en las horas de la noche usaban velas.

Consolidación física del asentamiento y clientelismo: el proceso de urbanización del asentamiento ha estado atravesado por la influencia de líderes políticos. Han sido recurrentes los escándalos de corrupción en la administración pública local. Muchos de los alcaldes y gobernadores son investigados o están presos por delitos emanados de su función. Algunos de los políticos que pasaron por Salvador Allende, basados en los relatos y registros

\footnotetext{
${ }^{16}$ Alfonso Torres Carrillo, "Identidades barriales y subjetividades colectivas en Santafé de Bogotá", Folios, n. ${ }^{\circ}$ 10 (2017), doi: https://doi.org/10.17227/01234870.10folios20.34.
} 
fotográficos de líderes, especialmente los aportados por Juan Bautista Morales, presidente de la JAC fueron:

Ancizar López López, cacique político liberal tradicional. Una de las personas que impulsó la creación del departamento del Quindío. Concejal y alcalde de Armenia, representante a la Cámara por el departamento de Caldas, senador y primer gobernador del Quindío. Manejó bastas clientelas políticas en la región y es mencionado por los pobladores más antiguos como uno de las figuras políticas que visitaban el lugar desde mediados de la década del 60.

David Barros Vélez, gobernador del Quindío designado 1986-1987, alcalde de Armenia 2004-2007, senador 1990-1991 y exnotario. En el año 2011 fue condenado a 17 años de prisión por peculado por apropiación, después de 20 años de trabajo político terminó en la cárcel por corrupción.

Luz Piedad Valencia, alcaldesa y aspirante al Senado por el Partido Liberal, hija de un cacique tradicional de la región, Luis Emilio Valencia Díaz, el Taita, quien ha dominado la política y la economía del Quindío. Protagonizó en el 2018 un escándalo que sacudió a la capital quindiana, fue sancionada por la Procuraduría General por cuenta de irregularidades en el recaudo y manejo de dineros de la valorización.

Si bien, los líderes comunitarios del lugar, históricamente, han recurrido a los políticos de turno para llevar progreso a la comunidad y solucionar algunas necesidades básicas insatisfechas de los pobladores, en los últimos años se ha presentado un giro en contra del electoralismo. Se capacitaron y aprendieron sobre el presupuesto público y los mecanismos de participación para acceder a recursos. Han generado un aprendizaje político autodidáctica movidos por la necesidad de entender normas, códigos y llevar a cabo la defensa de sus propios intereses comunitarios o individuales.

Aunque el hecho de ser un barrio informal dificulta la participación y la inversión, ante el panorama de corrupción, los líderes comprendieron que hay otras maneras de acceder a los recursos, aun cuando para ellos están prácticamente restringidos. La paradoja es que son invisibles para el gasto público, pero siguen siendo un caudal electoral potencial.

Respecto a la consolidación urbana, los procesos de autoconstrucción son típicos de los barrios que nacen a partir de las invasiones o posesión de terrenos particulares o del Estado. La vivienda atraviesa una serie de trasformaciones del material de construcción, que va desde lo transitorio (esterilla, guadua, zinc, plástico, cartón, alambre) a los materiales permanentes (ladrillo, cemento, arena, hierro). En los asentamientos, las personas están interviniendo constantemente sus viviendas para hacer pequeñas mejoras a la estructura, con ayuda, en ocasiones, de sus vecinos y familiares.

Organización social y amoblamiento comunitario: las primeras reuniones de la Junta de Acción Comunal se realizaban en las casas de los líderes comunitarios. Don Gustavo Parra, contó que la Junta comenzó siendo de la vereda Límites, en 1965 aproximadamente, pero debido a algunas divisiones e intereses se creó una nueva junta urbana. La primera caseta de acción comunal se construyó donde hoy está la capilla del sector, la actual queda en la mitad del barrio.

En agosto de 1988, la Junta de Acción Comunal del asentamiento obtuvo su personería jurídica, hoy cuenta con un archivo donde reposan los documentos legales de la gestión. La zona baja del asentamiento al independizarse (Regivit bajo) constituyó su propia Junta de Acción Comunal.

En el devenir del barrio fue clave el liderazgo ejercido por las madres comunitarias. Por esta razón, doña Elsa Reyes y otras mujeres del lugar propusieron organizar las primeras 
guarderías del sector. Esta gesta solidaria de origen popular contó con el apoyo de familiares y vecinos que ayudaron con su fuerza de trabajo a construir estos lugares. Muchas mujeres lograron de esta manera obtener un recurso económico extra para sus familias.

Una de las personas que guardan en su memoria los procesos de consolidación del barrio es don Gustavo Parra Delgado, hijo de don Campo Elías Parra, su padre fue llamado el patriarca del Salvador (Figura 2). Nos contó que a los 12 años llegó al lugar en compañía de su familia. Junto con su padre empezó a colonizar ciertas partes del norte de la ciudad, realizando amplios sembrados en la zona (plátano, yuca y café), en el sector donde hoy se encuentra el asentamiento Salvador Allende.

Don Gustavo nos compartió varios recortes de periódico con noticias sobre el asentamiento. Él y su familia fueron algunos de los primeros colonizadores de esta zona. También nos contó acerca de la ruta del tren y sobre cuestiones legales (propiedad y servidumbre según el eje de la vía férrea). Cuestiona por qué a otras personas que también construyeron sobre los rieles (en el centro de Armenia) les dieron papeles y a la gente que está hoy en el Salvador Allende se los niegan; para él debería existir el derecho a la igualdad, pues muchos ricos también se apropiaron de esos terrenos y a ellos si les legalizaron, afirmó.

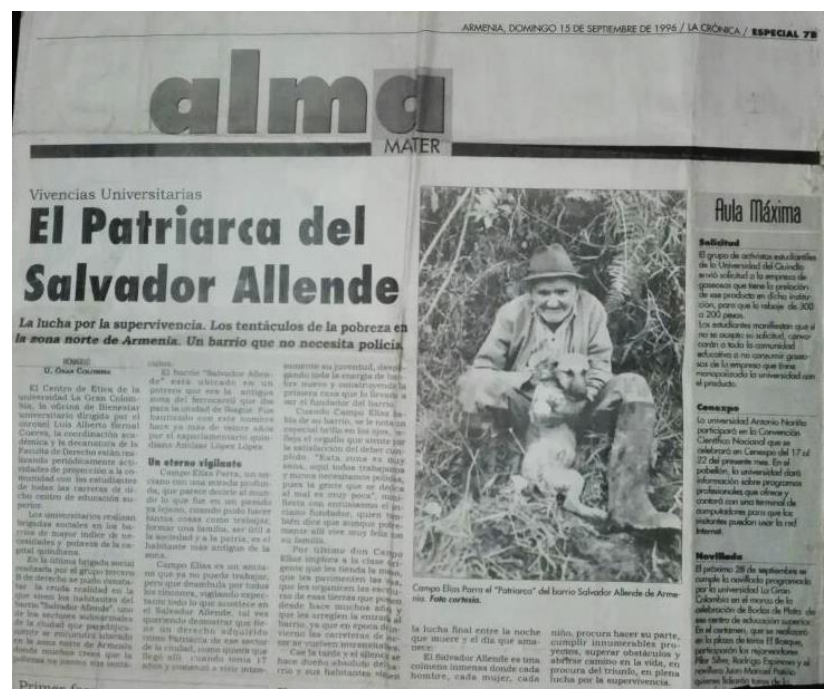

Figura 2. Artículo sobre Campo Elías Parra.

Fuente: Periódico La Crónica (Quindío), 15 de septiembre de 1996.

\section{El aniversario del asentamiento informal Salvador Allende}

El 16 de septiembre de 2017 se llevó a cabo la celebración del aniversario del asentamiento informal Salvador Allende ${ }^{17}$. Entre otras luchas, se conmemoró la vida, obra y muerte del presidente socialista Salvador Guillermo Allende Gossens, cuyo gobierno de la Unidad Popular fue derrocado un fatídico 11 de septiembre de 1973.

Si bien, todo esto apuntaría a la existencia de unas connotaciones políticas reivindicativas y a lecturas de la comunidad desde esta óptica, pocas de las personas entrevistadas sabían sobre este líder latinoamericano, solo algunos lograron explicar

\footnotetext{
${ }^{17}$ Los jóvenes del semillero participaron es este evento; de esta manera, contrastaron los conocimientos básicos, adquiridos en clase, con su contexto próximo (el lugar que habitan). Se estableció ese puente tan necesario (algunas veces inexistente) entre teoría y realidad. Y así, en campo utilizaron y entendieron el concepto de historia, pusieron en práctica los métodos de investigación social y del historiador.
} 
brevemente quién fue y qué hizo en su país. Por lo pronto, en el colegio, desde las clases de historia local, se abordan este y otros temas, como el origen de los nombres de los sectores aledaños e incluso de los municipios del Quindío, las luchas urbanas locales por acceso a la vivienda, el caso de la empresa Burila, etc.

En este sentido, no se puede afirmar que las personas que habitan el asentamiento Salvador Allende se asuman o lean su realidad en clave política y tampoco desde luchas sociales o reivindicativas de sus derechos.

Por esta razón, se intentó realizar un acercamiento a la historia del barrio, con el objetivo de lograr la apropiación de un saber histórico a partir de acciones colectivas con la JAC del asentamiento Salvador Allende y el Semillero de Investigación Cultivando Historia. Se programaron diversas actividades para la conmemoración del aniversario del lugar, como un primer paso en esta búsqueda de empoderamiento popular. Algunas de las actividades ejecutadas por el semillero fueron:

- Concursos "Crea el escudo, la bandera del barrio"- "La foto más antigua".

- Presentación del semillero SEICHI, "Conozcamos la historia de nuestro barrio".

- Sancocho comunitario, Miti-miti.

- Elaboración de cronologías a través del archivo fotográfico familiar.

Gracias a este espacio brindado por la JAC, los estudiantes pudieron socializar con sus vecinos y familiares los resultados de la investigación "Salvador Allende, una historia que contar". Compartieron datos de interés sobre la fundación, primeros pobladores, origen del nombre, llegada de los servicios públicos, vida cotidiana y perspectivas a futuro.

\section{Productos audiovisuales y socialización con la comunidad}

La historia tiene un papel político, hace personas críticas y propositivas, más sensibles y humanas. La historia está viva, pero se nos muere del letargo. Por ello, se propiciaron escenarios para la discusión y la democratización de la información, se buscó validar nuestro ejercicio con la gente y la comunidad, pues el propósito era transformar y mover sus fibras.

Por esta razón, los estudiantes del SEICHI realizaron un video donde se presentaron tanto los resultados de investigación como el proceso vivido por el colectivo en el quehacer del joven historiador e investigador ${ }^{18}$. EL video fue divulgado en la comunidad y entregado como material de consulta, no solo a la JAC, sino a la institución educativa, que además deberá tener una versión en su página web.

Este proceso de divulgación fue muy significativo, pues en él se vieron reflejados los estudiantes y vecinos del asentamiento Salvador Allende, protagonistas de su propia historia barrial a través de la memoria individual y colectiva. Siendo una oportunidad para pensar el territorio, los procesos sociales, culturales, económicos y políticos del lugar, su impacto y posibilidades. Como diría Freire: "Los hombres no se hacen en el silencio, sino en la palabra, en el trabajo, en la acción, en la reflexión"19.

\footnotetext{
${ }^{18} \mathrm{El} \quad$ video, $\quad$ puede ser consultado en el siguiente enlace: https://sites.google.com/view/semillerocultivandohistoria/p\%C3\%A1gina-principal.

${ }^{19}$ Paulo Freire, Pedagogía del oprimido (México: Siglo XXI editores, S.A., 1970), http://www.servicioskoinonia.org/biblioteca/general/FreirePedagogiadelOprimido.pdf.
} 
En la socialización de los resultados del proyecto de investigación, los padres de familia afirmaron que era muy importante conocer las raíces, la historia del barrio y a los vecinos, y expandir este tipo de investigación a otros barrios. Incluso, algunos sugirieron estudios históricos en diversas ramas, como género, medio ambiente, economía, entre otros.

\section{Conclusiones}

La implementación de este proyecto de investigación sobre historia barrial centró su trabajo en un semillero de investigación como herramienta didáctica alternativa para la formación de sujetos críticos de la historia local. En él, los jóvenes aprendieron y aplicaron técnicas de investigación social, con el fin de indagar sobre memoria histórica conservada y a la vez construida por vecinos, familiares y líderes del asentamiento informal Salvador Allende.

Se promovió de forma paralela y a través de la praxis, la enseñanza del "oficio del historiador" por parte del docente, se buscó que los estudiantes aprendiesen los pormenores de la producción de conocimiento en este campo del saber. Adicionalmente se suscitó el desarrollo del pensamiento reflexivo crítico a nivel social e histórico, pues se integraron las cuestiones socialmente vivas, abordadas desde los contextos de los estudiantes.

En palabras del Ministerio de Educación se busca que las y los estudiantes:

(...) afronten de manera crítica y creativa el conocimiento científico, tecnológico, artístico y humano que se produce (...) que comprendan la realidad nacional y desarrollen actitudes democráticas, responsables, tolerantes, solidarias, justas y éticas, se construyan como sujetos en y para la vida, comprendan la realidad nacional (pasado-presente) para transformar la sociedad en la que las y los estudiantes se desarrollen ${ }^{20}$.

Este proyecto logró estos fines de la educación al integrar no solo la teoría y la praxis de las ciencias sociales, sino al incorporar otras áreas. Desde la interdisciplinariedad se promovieron competencias de lecto-escritura y oralidad (elaboración de crónicas y otras narrativas digitales). Todo esto en pos del pensamiento reflexivo y crítico, la justa valoración del otro y de la memoria colectiva y el sentido de pertenencia por el barrio, evidenciado en la participación de los estudiantes en diversos escenarios de la vida comunitaria.

Respecto a los objetivos planteados, se puede afirmar que se cumplieron, pues se formó a los estudiantes en los métodos de investigación social y el oficio del historiador, aplicaron técnicas de investigación que les permitió dar cuenta de la historia barrial.

De igual manera, se llevó a cabo el análisis de la información, el contraste de las fuentes, la trascripción de entrevistas, la elaboración y presentación del video como informe final de investigación ante la comunidad educativa y la JAC del asentamiento informal Salvador Allende.

Por último, se llevó a cabo el proceso de análisis y redacción de las reflexiones en torno a los impactos del semillero de investigación como herramienta didáctica para la enseñanza de la historia y las ciencias sociales en educación media, así como su influencia en la formación de sujetos críticos de la historia local.

\footnotetext{
${ }^{20}$ Ministerio de Educación, "Lineamientos curriculares Ciencias Sociales" ..., 13.
} 


\section{Logros}

Los estudiantes que participaron en el proyecto, cambiaron positivamente su concepción sobre la Historia como disciplina, las formas de aprender, las relaciones con la comunidad y la investigación como ruta para descubrir y generar conocimiento. Para ellos la escuela puede ser según sus anhelos un escenario emocionante y más incluyente, alineado con sus necesidades, en el que se valoren sus aportes, sus preguntas, y sobre todo un espacio para recrear la democracia, el amor por el saber y por sus semejantes.

Los jóvenes afirmaron que en un semillero de investigación es posible: aprender, preguntar, escuchar, crear, compartir, divertirse, conocer a otras personas, tener consensos y desacuerdos, ser comprometidos, tener nuevos amigos, amar lo que se hace, saber que la opinión de cada integrante es importante y pueden aportar al trabajo colectivo, equivocarse, interpretar, analizar, ser dedicados, valorar el trabajo en equipo, reflexionar, saber apreciar la memoria histórica y tratar con respeto los conocimientos sobre el pasado y a las personas que los resguardan.

La formación en ciencias sociales, historia e investigación les permitió a los estudiantes hacer lecturas más profundas de la realidad y ejercer, desde su papel, un rol responsable, son a futuro ciudadanos más críticos y democráticos. Se encontró un sentido a lo estudiado en la escuela al contrastar teoría y realidad. Los estudiantes se apropiaron del territorio al recorrer y analizar sus componentes, aprendieron a apreciarlo, a verlo con otros ojos y a querer transformarlo para mejorar las condiciones sociales de su comunidad.

Se conocieron diversas instituciones y personas importantes en el campo de la historia y la investigación en la ciudad, entre ellas:

El Centro de Documentación Regional en Arqueología y Planeación Territorial, del Museo del Oro Quimbaya del Banco de la República y su analista cultural (cuyo trabajo es contribuir en la orientación a procesos de investigación dentro y fuera de la sucursal).

La biblioteca de la Universidad del Quindío y su centro de documentación (especializado en archivos posterremoto). El director del lugar les brindó una charla a los estudiantes sobre los archivos y los felicitó por interesarse en la historia de la ciudad. Les enseñó las herramientas de consulta 2.0. y los invitó a seguir sus sueños y vincularse a la universidad a través de las diferentes carreras profesionales.

Los fundadores de su barrio, líderes comunitarios, vecinos y profesores, a quienes reconocieron como parte importante en la consolidación del barrio y el proceso de escritura de su historia. Comprendieron la importancia de las historias personales y cómo éstas alimentan la historia general.

Otros logros importantes fueron la relación de los contenidos del plan de área de ciencias sociales con el proyecto de investigación y con la vida comunitaria, lo cual les permitió a los estudiantes establecer puentes entre la historia y la vida barrial, participaron en la conmemoración del aniversario del asentamiento. Los estudiantes autogestionaron el conocimiento, investigaron y preguntaron sobre el tema, consiguieron información secundaria, gestionaron contactos e interactuaron con personas de su comunidad concertando citas para entrevistas y aportaron datos adicionales que consiguieron al socializar con vecinos la propuesta de trabajo.

Los estudiantes prestaron su servicio social obligatorio en una actividad intelectual y productiva (investigación), al menos en la primera etapa del proceso. Se sistematizó la experiencia a partir de la creación de material audiovisual y literario sobre el tema, el cual fue socializado con la comunidad educativa y el barrio. Se le apostó a la transversalidad en el proceso de investigación. 
Los estudiantes lograron acercarse al conocimiento como científicos sociales, conocieron el método científico y lo vivieron en el proceso de investigación, llevándolo a su contexto inmediato. Le otorgaron contexto y función al conocimiento de lo social.

La visita a museos, centros de documentación, bibliotecas, entre otros paisajes históricos, enseñó a los jóvenes que el pasado se evidencia de manera física y no escapa de ser destruido por desconocimiento de propietarios, funcionarios públicos o ideas de modernización que arrasan con las construcciones y con formas antiguas de la arquitectura local. Los estudiantes adquirieron una actitud responsable respecto al patrimonio cultural de la ciudad, para conocerlo y protegerlo. Reconocieron la memoria barrial y local como hilos del pasado que nos permiten contextualizar los hechos del presente.

Se obtuvo el interés de los estudiantes, líderes comunitarios y padres de familia para que el proceso trascienda, y que la experiencia no se quede solo como un ejercicio escolar.

\section{Problemáticas}

El escaso apoyo institucional por parte de la directiva del colegio a las propuestas pedagógicas alternativas, los diversos obstáculos administrativos y académicos que tuvieron que ser resueltos desde instancias superiores como el Ministerio de Educación Nacional (Programa Becas para Excelencia Docente) y la Secretaría de Educación Municipal de Armenia a favor del proyecto de investigación formativa.

Desde el punto de vista pedagógico, la implementación de la propuesta permitió identificar un evidente choque de las viejas estructuras de enseñanza (modelos implementados y defendidos con vehemencia por algunos colegas y directivos, los cuales responden al enfoque conductista, centrados en la nota y su carácter cuantitativo como resultado del proceso educativo), contra propuestas críticas, menos homogeneizantes y más tendientes a potencializar habilidades desde la investigación acción. Pasará seguramente mucho tiempo antes de que puedan ejecutarse sin sospecha nuevos emprendimientos en este campo, lo cual es una lástima, pues se ha comprobado en diferentes ocasiones sus buenos resultados.

Limitaciones respecto a infraestructura, inexistencia de escritorios y espacios adecuados para el trabajo documental. El establecimiento educativo no cuenta con sala de sistemas, sala de lectura en la biblioteca ni aula máxima y no hay salones disponibles en las horas de la tarde (por el cumplimiento de la jornada única).

Los semilleros de investigación requieren la inversión de un tiempo adicional por parte del docente y de los estudiantes para las actividades de planeación y programación, las salidas de campo, lecturas y redacción, preparación de actividades por parte del docente para los escenarios de clase y campo, gestión de permisos, charlas y visitas, entre otros. Si no se cuenta con el apoyo de los rectores, no es fácil mantener en el tiempo este tipo de procesos; por ejemplo, la prestación de servicio social a los estudiantes compensaría el trabajo extraclase que ellos realizan (durante el 2018 fue negado este beneficio). Así mismo, en varias ocasiones se les negó al docente y estudiantes asistir a reuniones, salidas pedagógicas, seminarios y foros, en horarios escolares.

No hay disponibilidad de recursos económicos institucionales para el desplazamiento a museos, centros de documentación, bibliotecas, monumentos, entre otros. Así pues, en este caso, los gastos corrieron por cuenta del docente y se tuvo que marginar a muchos estudiantes de este tipo de actividades. 
Son crecientes las actividades burocráticas en la escuela, razón por la cual es difícil formarse rigurosamente y dedicarse a temas de historia local, además por la responsabilidad que se debe asumir en proyectos institucionales administrativos y cátedras transversales.

Ante los obstáculos mencionados, se efectuaron empalmes con iniciativas de investigación y memoria en otros colegios. Actualmente, se está montando una galería de fotografía sobre memoria histórica, retomando las cuestiones socialmente relevantes (todo esto en horario extraclase con permiso de acudientes).

Estas prevenciones y dificultades se pueden presentar en general en el desarrollo de la investigación educativa en otros contextos, sin embargo, deben ser asumidos como un impulso para llevar adelante las propuestas. En el caso del semillero Cultivando Historia, el impacto se ratificó con el apoyo de los líderes comunitarios, los padres de familia de los estudiantes del grado 10-A y los integrantes del Semillero durante los años 2017 y 2018. También fue decisivo el apoyo de la comunidad del asentamiento Salvador Allende y su Junta de Acción Comunal, quienes manifestaron su deseo de replicar la experiencia a través de la Junta Administradora Local.

\section{Recomendaciones}

Por último, como afirma Nietzsche en sus consideraciones intempestivas y de la utilidad y los inconvenientes de la historia para la vida:

Necesitamos la historia para la vida y la acción, no para apartarnos cómodamente de la vida y la acción, y menos para encubrir la vida egoísta y la acción vil y cobarde. Tan solo en cuanto la historia está al servicio de la vida queremos servir a la historia ${ }^{21}$.

No se debe caer en una enseñanza anticuaria de la historia. Algo que podría pasar si nos ocupamos solo de retazos de la historia local, entregándolos como datos aislados, sin enlazarlos con los referentes previos de los estudiantes, sin que los analicemos en relación con un contexto o importancia para el curso. Por eso, la investigación escolar es un camino integrador y una gran herramienta didáctica para la enseñanza actual de la historia local.

Este trabajo, puede servir como referente para nuevas investigaciones que se desarrollen en el campo de las didácticas alternativas, relacionadas esencialmente con la investigación formativa como herramienta de enseñanza y aprendizaje, desde el área de las ciencias sociales y en especial desde la historia local. Podrá ser útil para aquellos que valoren la generación de conocimiento a partir de colectivos de investigación y pensamiento que trascienden las áreas del saber.

Puede ser útil para investigaciones que se desarrollen desde la pedagogía crítica, y que involucren la participación y empoderamiento de los estudiantes, tendientes a la trasformación de su realidad social y/o a las condiciones de vida de su comunidad. Prácticas como la implementación de semilleros de investigación en la educación media, pueden suscitar líneas de acción investigativas en didácticas que aborden el proceso de enseñanza fuera del aula, con el uso de la ciudad, sus instituciones y elementos constitutivos como insumo para un aprendizaje que trasciende barreras físicas y mentales.

\footnotetext{
${ }^{21}$ Friedrich Nietzsche, Sobre la utilidad y los perjuicios de la historia para la vida (Madrid: Editorial EDAF, 2000), https://books.google.com.co/books?id=IlEl4HBYxHcC\&pg=PA32\&lpg=PA32\&dq.
} 
Para terminar, puede ser útil para aquellos docentes que día a día en el buen sentido de la palabra "cuestionan su labor", porque saben que la pregunta es la ruta para generar nuevo conocimiento, seguros de que no todo está dado y que nuestra profesión y vida son proyectos inacabados, comprometidos y sinceros que ven la investigación con buenos ojos y sin prevenciones.

La historia no es lo que se sabe, sino lo que se busca. Por ello, como reflexión final cierro con estas palabras de la experiencia:

Con cada visita a don Salvador (porque, con el pasar de las horas, al ser partícipe del cariño de los vecinos, el barrio adquirió carácter, una presencia de viejo trabajador con sombrero y pantalón raído, hombre pobre pero digno, sonriente y soñador) se tuvo el deseo sincero de impactar positivamente a la institución educativa y a la comunidad. Y siempre se vio el objeto de estudio tan al norte, tan discordante con sus casuchas de techos improvisados, contrastadas con los edificios imponentes de las gentes acomodadas, tan alto como el nevado que se deja ver en las mañanas despejadas. Esas fueron y serán la inspiración, los estudiantes y sus familias. Siempre, usar una forma que integre lo académico con lo real y palpable (para mostrar otra cara del proceso educativo fuera de las aulas) e intentar cambiar el imaginario colectivo de una comunidad cuyo concepto es desfavorable para muchos.

Este proceso, este semillero de investigación, este "hijo" (de todos), es un intento por visibilizar a los subalternos, que no solo son invisibles en la vida administrativa de la ciudad, sino del paisaje y de la vista de quienes la habitan.

Esto nos llevó a hacer apuestas por las prácticas alternativas pedagógicas, a la implementación del semillero de investigación, a la creación de colectivos de memoria, a la transversalidad desde las diferentes áreas, a dinamizar la enseñanza y el aprendizaje de las ciencias sociales desde la implementación del oficio de historiador como estrategia didáctica de la investigación formativa.

Este proyecto no solo buscó aportar en la emancipación y liberación de la mente de los estudiantes y habitantes de un asentamiento informal. Este proyecto quiso transitar y habitar el territorio, para apropiárselo, transformarlo y devolver la voz a los vencidos, a aquellos que no tuvieron amanuenses, pero guardan intacta la memoria personal y de su comunidad. Y a los subalternos, aquellos que, como nosotros, luchan cada día por sus derechos y su dignidad. A quienes con honor reviven las palabras de Salvador Allende: "La historia es nuestra y la hacen los pueblos", y por esta razón merece la pena ser contada. 


\section{Bibliografía}

Carr, Edward Halle. ¿Qué es la historia? (Conferencias, "George Macaulay Trevelya" dictadas en la Universidad de Cambridge. Enero - marzo de 1961). Universidad de Cambridge: $\quad$ Editorial Ariel, https://introduccionalahistoriaunlp.files.wordpress.com/2014/03/carr-que-es-lahistoria-cap1-el-historiador-y-los-hechos.pdf.

Clavijo Vélez, Sandra Catalina. "Características físicas y sociales de los asentamientos informales de Armenia en los años 1997- 2008”. Tesis de pregrado. Universidad del Quindío, 2008.

Correa Ramírez, Jhon Jaime. "Historia local: el ritmo de la historia barrial". Antropología y Sociología: Virajes (2006): 203-223.

Departamento Administrativo de Planeación. Diagnóstico Local Participativo. CODELPA. Comité de desarrollo local participativo de Armenia, 2016. http://planeacionarmenia.gov.co/comuna-10/.

Freire, Paulo. Pedagogía del oprimido. México: Siglo xxi editores, S.A., 1970. http://www.servicioskoinonia.org/biblioteca/general/FreirePedagogiadelOprimido.pdf

Friedrich Nietzsche. Sobre la utilidad y los perjuicios de la historia para la vida. Madrid: Editorial EDAF, 2000 . https://books.google.com.co/books?id=IlEl4HBYxHcC\&pg=PA32\&lpg=PA32\&dq.

Ley 115/1994, del 8 de febrero. Ley general de educación. Diario Oficial No. 41.214. República de Colombia. https://www.mineducacion.gov.co/1621/articles85906_archivo_pdf.pdf.

Martínez Rincón, Hernán. "El puente de Boquía”. Acceso el 15 de mayo de 2019. http://academiadehistoriadelquindio.blogspot.com/2014/07/el-puente-de-boquia.html.

Ministerio de Educación Nacional, "Lineamientos curriculares Ciencias Sociales" (2002). https://www.mineducacion.gov.co/1621/articles-89869_archivo_pdf.pdf.

Ramírez Ospina, Duván Emilio. “Capital humano como factor de crecimiento económico: caso departamento de Caldas (Colombia). 1983 2003”. Acceso el 15 de mayo de 2019. http://www.eumed.net/libros-gratis/2007b/271/4.htm.

Sánchez Puentes, Ricardo. Enseñar a investigar: Una didáctica nueva de la investigación en ciencias sociales y humanas. Universidad Nacional Autónoma de México: Instituto de Investigaciones sobre la Universidad y la Educación, 2014. http://disde.minedu.gob.pe/bitstream/handle/123456789/4635/Ense\%C3\%B1ar\%20a \%20investigar\%20una\%20did\%C3\%A1ctica\%20nueva\%20de\%201a\%20investigaci 
$\% \mathrm{C} 3 \% \mathrm{~B} 3 \mathrm{n} \% 20 \mathrm{en} \% 20 \mathrm{ciencias} \% 20$ sociales $\% 20 \mathrm{y} \% 20$ humanidades.pdf?sequence $=1 \&$ isAllowed $=\mathrm{y}$.

SCRIBD. "Enseñanza para la Comprensión (Tomado de ALPS de la Universidad de Harvard en http://learnweb.harvard.edu)". Acceso el 27 de mayo de 2019. https://www.scribd.com/document/336645771/Comprension-Metas-DesempenosValoracion.

Thompson, Paul. "Historia oral y contemporaneidad". Anuario $N^{\circ} 20$ - Escuela de Historia $\begin{array}{llllllll}- & F H & y & A & - & \text { UNR } & \text { (2017). }\end{array}$ www.anuariodehistoria.unr.edu.ar/ojs/index.php/Anuario/article/download/204/223.

Torres Carrillo, Alfonso. Hacer historia desde abajo y desde el sur. Bogotá: Ediciones desde abajo, 2014. https://www.academia.edu/32287760/Hacer_historia_desde_abajo_y_desde_el_Sur.

. "Identidades barriales y subjetividades colectivas en Santafé de Bogotá". Folios, n. 10 (2017): 24-34. doi https://doi.org/10.17227/01234870.10folios20.34. 